\title{
THE RETURN OF THE ANOMALOUS COSMIC RAYS TO 1 AU IN 1992
}

\author{
R. A. Mewaldt, A. C. Cummings, J. R. Cummings, and E. C. Stone \\ California Institute of Technology \\ B. Klecker, D. Hovestadt, and M. Scholer \\ Max-Planck-Institut für Extraterrestrische Physik \\ G. M. Mason, J. E. Mazur, and D. C. Hamilton \\ University of Maryland \\ T. T. von Rosenvinge \\ Goddard Space Flight Center
}

J. B. Blake

Aerospace Corporation

\begin{abstract}
New observations of low energy $(\sim 1$ to 200 $\mathrm{MeV} / \mathrm{nuc}$ ) cosmic rays measured by three newly launched experiments on SAMPEX during 1992 and 1993 show the strong presence of anomalous cosmic ray (ACR) nitrogen and oxygen, well before the approaching solar minimum. When compared with ACR temporal variations over the past two solar cycles we find that the 1992-1993 fluxes are -5 to 10 times their level at corresponding neutron monitor counting rates in 1969-1970 and 1985.
\end{abstract}

\section{Introduction}

It is now twenty years since the discovery of anomalous flux increases in the low energy ( $<50 \mathrm{MeV} / \mathrm{nuc}$ ) spectra of helium and oxygen led to the identification of a new component of cosmic rays (McDonald et al. 1974, GarciaMunoz, Mason, and Simpson 1973, Hovestadt et al. 1973). This "anomalous" cosmic ray (ACR) component is now thought to represent interstellar neutral particles that have drifted into the heliosphere, been singly-ionized by solar UV or by charge exchange with the solar wind, convected into the outer heliosphere, and then accelerated to energies of 10 to $100 \mathrm{MeV} /$ nuc (Fisk, Kozlovsky, and Ramaty 1974), probably at the solar wind termination shock (Pesses, Jokipii, and Eichler 1981). The ACR component is especially sensitive to solar modulation, varying over the solar cycle by a factor of $>100$, and observable at $1 \mathrm{AU}$ only at solar minimum. The elements whose energy spectra in the outer heliosphere exhibit anomalous increases in flux above the low energy galactic cosmic ray (GCR) spectrum include $\mathrm{He}$, $\mathrm{C}, \mathrm{N}, \mathrm{O}, \mathrm{Ne}, \mathrm{Ar}$, and possibly $\mathrm{H}$ (see, e.g., Cummings and Stone 1988; Christian, Cummings, and Stone 1989), although only $\mathrm{He}, \mathrm{N}, \mathrm{O}$, and $\mathrm{Ne}$ are clearly identifiable at $1 \mathrm{AU}$.

In this paper we present observations of $\mathrm{C}, \mathrm{N}$, and $\mathrm{O}$ covering nearly three decades in energy/nuc from the newlylaunched Solar, Anomalous, and Magnetospheric Particle Explorer (SAMPEX). These observations indicate that during the current recovery to solar minimum the ACR component returned to $1 \mathrm{AU}$ (became observable) in mid-1992, well before it might have been expected to on the basis of its behavior during previous solar cycles, and that it is already

\section{Copyright 1993 by the American Geophysical Union.}

Paper number 93GL02493

0094-8534/93/93GL-02493\$03.00 approaching flux levels observed during previous solar minima. A preliminary report of some of the data reported here appears in Mewaldt et al. (1993).

\section{Instrumentation}

The SAMPEX spacecraft was launched in July 1992 into an $82^{\circ}$ inclination, low Earth orbit $(670 \times 520 \mathrm{~km})$ carrying four instruments that measure ion and electron fluxes over a broad range of energy and intensity, including particles of solar, interplanetary, galactic, and mag-netospheric origin (Baker et al. 1993). Measurements from three of these instruments are combined here to provide a comprehensive determination of interplanetary cosmic ray spectra for the period from late 1992 to early 1993.

The Max-Planck-Institut/Aerospace Corp. Heavy Ion Large Telescope (HILT) covers the primary ACR energy range with a very large geometry factor $\left(60 \mathrm{~cm}^{2} \mathrm{sr}\right)$ that provides accurate determinations of the low ACR fluxes in a short time interval. The Caltech/Goddard Mass Spectrometer Telescope (MAST) is optimized for isotopes of energetic nuclei from $\mathrm{C}$ to $\mathrm{Ni}(\mathrm{Z}=6$ to 28$)$, and extends spectral measurements to higher energy than HILT. The University of Maryland Low Energy Ion Composition Analyzer (LEICA) covers the energy range below a few MeV/nuc. It is used to determine the lowest portion of the ACR spectrum and to monitor solar activity that might contaminate the quiet time spectra. Detailed descriptions of these instruments have been published elsewhere (Klecker et al. 1993; Cook et al. 1993; Mason et al. 1993). In addition, data from the Caltech experiments on IMP-7 \& 8 have been used to trace the ACR intensity over the twenty years before the SAMPEX launch.

\section{Observations}

The time periods used for this study were selected by monitoring the daily average flux of 0.6 to $1.25 \mathrm{MeV} /$ nuc He measured by LEICA at invariant latitudes $>70^{\circ}$. Included were those days for which this flux was $<0.01$ $\left(\mathrm{cm}^{2} \mathrm{sr} \cdot \mathrm{sec} \cdot \mathrm{MeV} / \mathrm{nuc}\right)^{-1}$. Figures 1 and 2 show quiet-time energy spectra for $C, N$, and $O$ measured from 16 November 1992 to 30 April 1993. Note that at energies $>50 \mathrm{MeV} /$ nuc the $\mathrm{C} / \mathrm{O}$ ratio is $\sim 1$, typical of galactic cosmic rays. Below $\sim 30 \mathrm{MeV} /$ nuc the $\mathrm{O}$ flux increases sharply, while the $\mathrm{C}$ flux continues to decrease, consistent with a GCR component subject to solar modulation, until it reaches $\sim 20 \mathrm{MeV} / \mathrm{nuc}$, where both HILT and MAST observe a turn-up in the C 


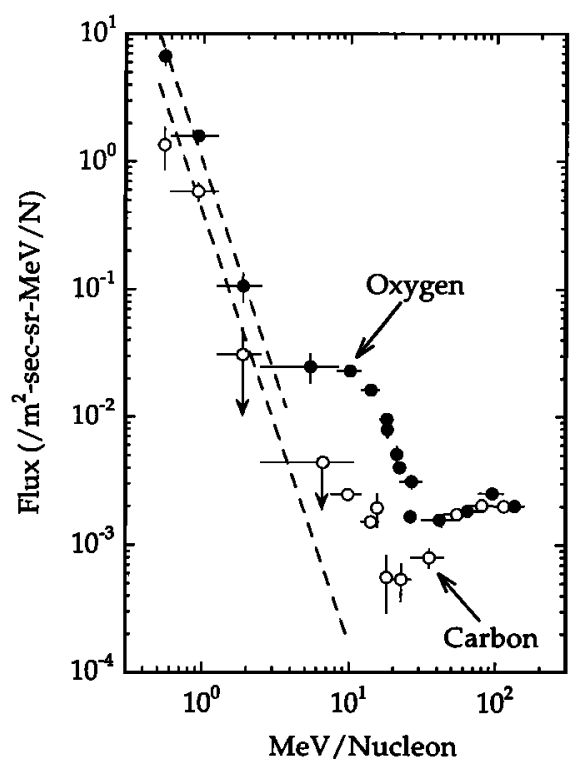

Fig. 1: Quiet-time spectra for $\mathrm{C}$ and $\mathrm{O}$ measured by SAMPEX at invariant latitudes $>70^{\circ}$ from $11 / 16 / 92$ to 4/30/93. The dashed line through the $O$ points is a fit to the 0.5 to $2 \mathrm{MeV} / \mathrm{nuc}$ data; the $\mathrm{C}$ line is derived from this assuming $\mathrm{C} / \mathrm{O}=0.4$, typical of low energy solar particles.

spectrum. At the lowest energies ( $<5 \mathrm{MeV} / \mathrm{nuc}$ ) there is evidence for a solar/interplanetary component.

Figure 2 shows that there is also an enhancement in the $\mathrm{N}$ spectrum below $\sim 35 \mathrm{MeV} /$ nuc; a similar turn-up is observed for $\mathrm{Ne}$ at this time (Mewaldt et al. 1993). The $\mathrm{Mg}, \mathrm{Si}$, and $\mathrm{Fe}$ spectra show no significant enhancements in the $\sim 10$ to 40 $\mathrm{MeV} /$ nuc energy range. The observed features in the $\mathrm{N}$ and $\mathrm{O}$ spectra are the classic signatures of anomalous cosmic rays, although it had not been generally expected that ACRs would be evident at $1 \mathrm{AU}$ this long before solar minimum.

Both HILT and MAST observe a small C enhancement at $\sim 10$ to $15 \mathrm{MeV} /$ nuc. Quiet time measurements at $1 \mathrm{AU}$ during previous solar cycles have sometimes observed small enhancements in the $\mathrm{C}$ spectrum at $\sim 10 \mathrm{MeV} /$ nuc (e.g., Webber et al. 1977), usually interpreted as due to small solar or interplanetary events that were not eliminated by the quiettime selection criteria. The only clear evidence for $\mathrm{ACR}$ carbon comes from Voyager observations in the outer heliosphere, where solar contributions are much less significant (Cummings and Stone 1988). The Voyager 10 to $20 \mathrm{MeV} /$ nuc $\mathrm{C} / \mathrm{O}$ ratio is $\sim 0.01$, whereas the ratio in Figure 1 is closer to 0.1 . While it is therefore possible that some of the "bump" in the 10 to $20 \mathrm{MeV} /$ nuc C spectrum is due to ACR carbon, there is more likely a significant solar contribution, and this region of the spectrum will require additional study as more SAMPEX data are obtained.

To relate the 1992-93 observations to those from earlier years Figure 3 shows the 8 to $27 \mathrm{MeV} /$ nuc oxygen flux over two solar cycles, based mainly on 1972 to 1992 data from the Caltech experiments on IMP-7\&8 (Mewaldt et al. 1976) supplemented by OGO- 5 and IMP- 6 data from earlier years, and extended with new data from SAMPEX. It has previously been shown that the ACR intensity is wellcorrelated with neutron monitor measurements of the flux of high-energy cosmic rays (Mewaldt, Stone, and Vogt 1975; von Rosenvinge and McDonald 1975; Klecker et al. 1980), and Figure 3 includes the Mt. Washington neutron monitor counting rate, taken to the 30 th power and normalized to the 1972 to 1978 ACR fluxes (Mewaldt 1990).

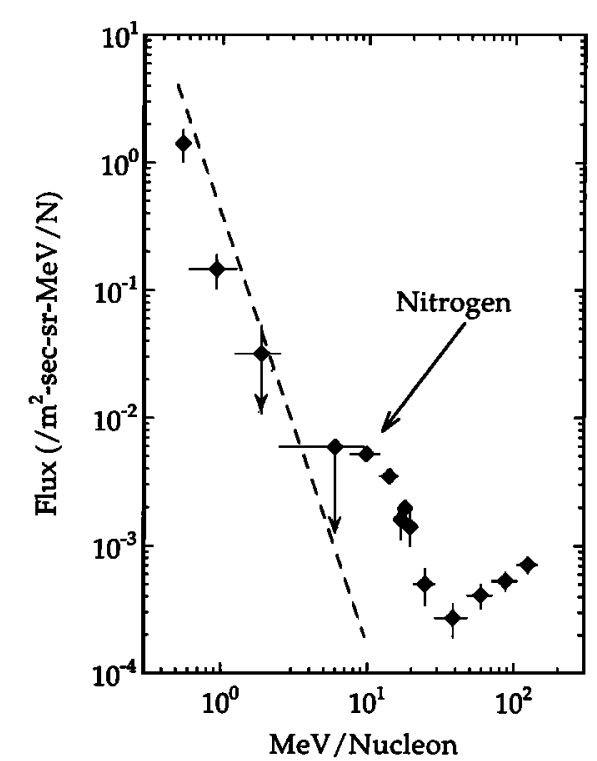

Fig. 2: Quiet time spectra for $N$ measured by SAMPEX under the same conditions as for Figure 1 . In Figure 1 and 2 data points from LEICA extend from $\sim 0.5$ to $10 \mathrm{MeV} /$ nuc; those from HILT start at $\sim 8$ and extend to $16 \mathrm{MeV} /$ nuc for $\mathrm{C}$ and $28 \mathrm{MeV} /$ nuc for O; while those from MAST extend from $\sim 15$ to $\sim 150 \mathrm{MeV} / \mathrm{nuc}$. The dashed line is derived from the $\mathrm{O}$ fit assuming $\mathrm{N} / \mathrm{O}=0.15$.

\section{Discussion}

While the scaling of the neutron monitor rates in Figure 3 provides a reasonable representation of the ACR temporal variations at $1 \mathrm{AU}$ over two solar cycles, the correlation is not perfect. Note that the 1987 solar minimum fluxes never quite reached their 1976-77 levels, even though the 1987 neutron monitor exceeded its $1976-77$ level. It is possible that this is a result of the differing effects of gradient and curvature drifts (e.g., Pesses, Jokipii, and Eichler, 1981) in the two orientations of the solar magnetic field, or it may be an example of "hysteresis" between low-energy and higher energy particles, in which the $1987 \mathrm{ACR}$ fluxes at $1 \mathrm{AU}$ never recovered to an equilibrium level before the onset of increased solar modulation in mid-1987.

A much greater difference between the ACR fluxes and scaled neutron monitor rate is evident in 1992, when the O flux is at least 5 times greater than would be predicted by the correlation extrapolated from earlier years. We might expect the 1992-1993 recovery to mimic its behavior 22 years earlier more closely than $\sim 11$ years earlier (e.g., Jokipii and Thomas 1981), since the solar magnetic field orientations are the same in these two cases. To examine the onset of these three successive solar minima in more detail, we show in Figure 4 regression plots between the ACR oxygen flux and the neutron monitor for the periods 1968 to 1972,1985 to 1987 , and 1992 to 1993. When compared at corresponding neutron monitor levels we see that the ACR oxygen flux at the beginning of 1993 is $~ 5$ times greater than in 1968-72. A similar low-energy increase is seen in the $N$ (Figure 2) and Ne spectra (Mewaldt et al. 1993).

The origin of the rapid recovery of ACR N and O in 1992 is presently unknown, but it may be related to a decrease in the tilt of the heliospheric current sheet (Cummings, Stone, and Webber 1990), which decreased from $>50^{\circ}$ in late 1991 to $<30^{\circ}$ in mid-1992 (Hoeksema, private communication). Corresponding current sheet data from 1969 to 1970 are not available, but the tilt in 1971-72 was already $<30^{\circ}$ in early 


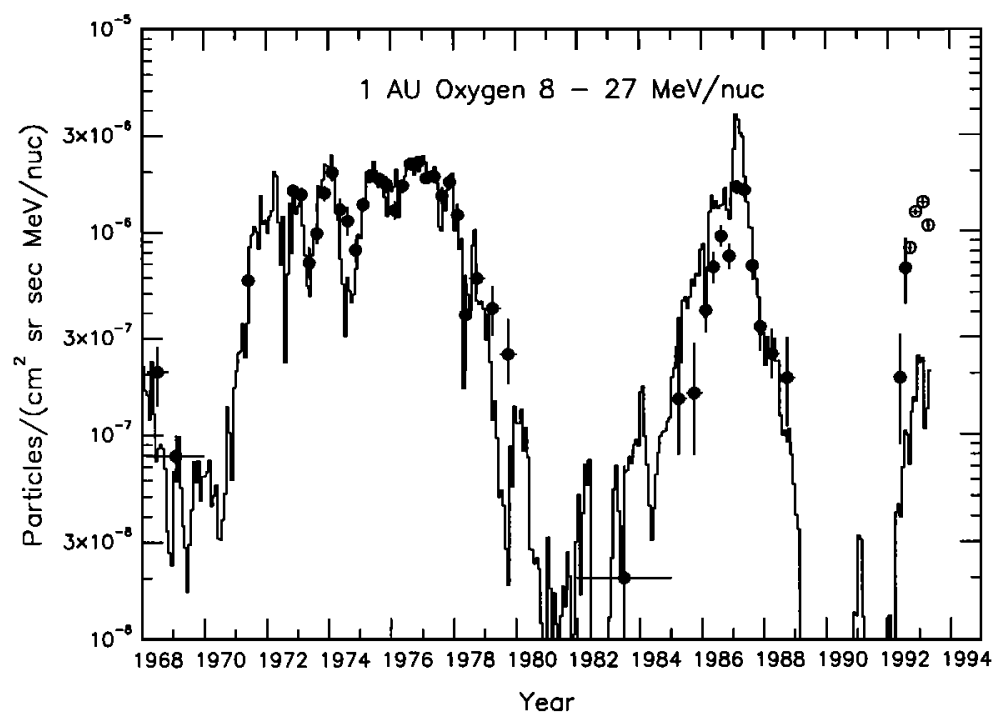

Fig. 3: The 8 to $27 \mathrm{MeV} /$ nuc oxygen flux from 1968 to early 1993, based mainly on 1972 to mid-1992 data from the Caltech experiments on IMP-7\&8. The 1968 (Teegarden et al. 1969) and 1968-69 (Mogro-Campero et al. 1973) points are from OGO5, while the 1971 point is from IMP-6 (von Rosenvinge and McDonald 1975). The last four (open) points in 1992-1993 are from the HILT sensor on SAMPEX. Also shown is the Mt. Washington neutron monitor rate (MtW; Labonte and Lockwood, private communication), scaled using the equation $\mathrm{J}=2.1 \times 10^{-6}(\mathrm{MtW} / 2440)^{30}$.

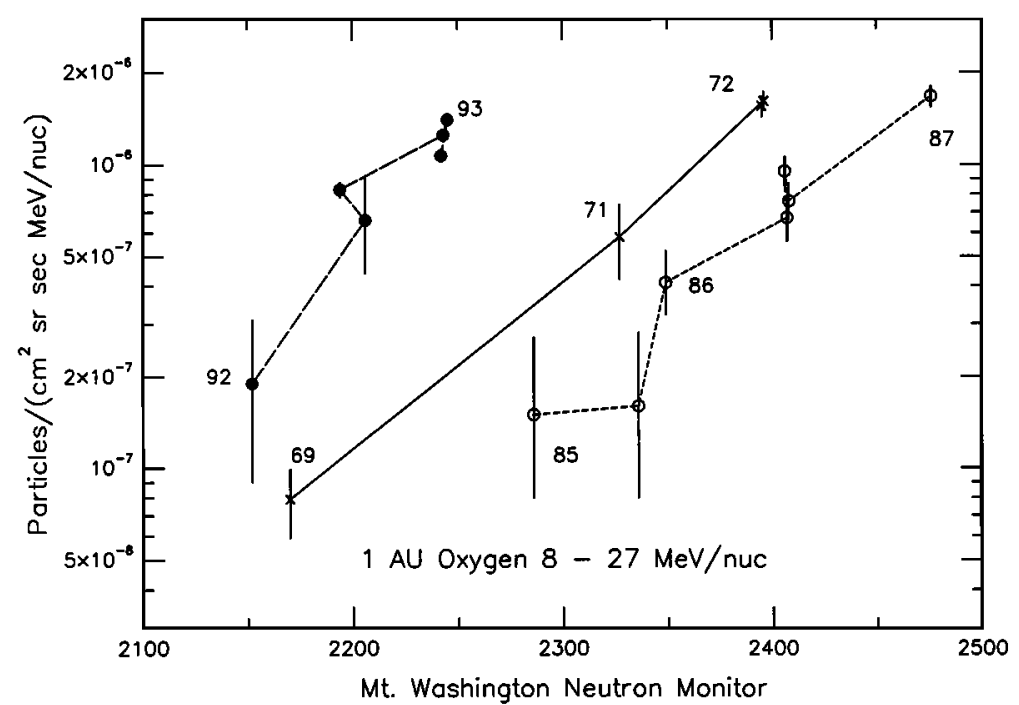

Fig. 4: Regression plots of the 8 to $27 \mathrm{MeV} /$ nuc oxygen flux vs. the Mt. Washington neutron monitor counting rate for the three recovery periods in Figure 3.

1971 (Saito and Swinson, 1986). The lack of current sheet (and detailed ACR) measurements from 1969-70 prevent us from exploring this possibility further.

It is also possible that the increased ACR flux at $1 \mathrm{AU}$ in 1992-1993 results in part from a closer approach of the solar wind termination shock than its corresponding distance in the early 1970's. We might expect the termination shock distance at any given time to reflect the solar wind pressure at $1 \mathrm{AU}$ $\sim 1$ year earlier. This possibility is under investigation, but it does not appear likely that a closer approach of the termination shock would affect the intensity at $1 \mathrm{AU}$ by as much as a factor of $\sim 5$, given the generally small intensity gradients measured in the outer heliosphere (Cummings and Stone 1988, Cummings, Stone, and Webber 1990).

It is also possible that the apparently different modulation of low and high energy cosmic rays in 1992 and the early 1970 's reflects a property of the solar wind and interplanetary magnetic field that determines the interplanetary diffusion coefficients of these two components, which depend on the power spectrum of magnetic field fluctuations in the interplanetary medium. In addition, a change in the diffusion coefficient in the vicinity of the termination shock might alter the ACR "source" strength.

Irrespective of its origin, the sudden return of the ACR fluxes to $1 \mathrm{AU}$ in 1992 provides a fortunate opportunity for the new instrumentation on SAMPEX to make improved measurements of its elemental and isotopic composition, charge states, temporal behavior, and its trapping in the Earth's magnetosphere (Cummings et al. 1993).

Acknowledgments. We are grateful to J. Labonte and J. Lockwood for providing unpublished Mt. Washington neutron monitor data and to J. T. Hoeksema for providing unpublished data on the neutral sheet tilt. This work was 
supported by NASA under contract NAS5-30704, grant NAGW-1919, and NASA Cooperative Agreement 26979B at The Aerospace Corporation. Work at the Max-Planck Institut was funded by the Bundesministerium fur Forschung und Technologie, FRG, under contract 50 OC 90021.

\section{References}

Baker, D. N., et al., An overview of the Solar, Anomalous, and Magnetospheric Particle Explorer (SAMPEX) mission, IEEE Trans. Geosci. Remote Sensing 31, 531, 1993.

Cook, W. R., et al., MAST: A Mass Spectrometer Telescope for studies of the isotopic composition of solar, anomalous, and galactic cosmic ray nuclei, IEEE Trans. Geosci. Remote Sensing 31, 557, 1993.

Christian, E. R., A. C. Cummings, and E. C. Stone, Evidence for anomalous cosmic ray hydrogen, Astrophys. J. 334, L77, 1988.

Cummings, A. C., and E. C. Stone, Composition, gradients, and temporal variations of the anomalous cosmic-ray component. Proc. Sixth Internat. Solar Wind Conference (Boulder), NCAR Technical Note 306 2, 599, 1988.

Cummings, A. C., E. C. Stone, and W. R. Webber, Time variation of radial and latitudinal gradients of anomalous cosmic ray oxygen in the outer heliosphere, Proc. $21 \mathrm{st}$ Internat. Cosmic Ray Conf. (Adelaide) 6, 190, 1990.

Cummings, J. R., et al., New evidence for geomagnetically trapped anomalous cosmic rays, Geophys. Res. Letr., Sept. 15, 1993.

Fisk, L. A., B. Kozlovsky, and R. Ramaty, An interpretation of the observed oxygen and nitrogen enhancements in low-energy cosmic rays, Astrophys J. 190, L35, 1974.

Garcia-Munoz, M., G. M. Mason, and J. A. Simpson, A new test for solar modulation theory: the 1972 May-July low energy galactic cosmic ray proton and helium spectra, Astrophys. J. 182, L81, 1973.

Hovestadt, D. O., et al., Differential energy spectra of lowenergy ( $<8.5 \mathrm{MeV}$ per nucleon) heavy cosmic rays during solar quiet times, Phys. Rev. Lett. 31, 650, 1973.

Jokipii, J. R., and B. Thomas, Effects of drift on the transport of cosmic rays IV. Modulation by a wavy interplanetary current sheet, Astrophys. J. 243, 1115, 1981.

Klecker, B., et al., On the charge state of the anomalous oxygen component, Geophys. Res. Lett. 7, 1033, 1980.

Klecker, B., et al., HILT: A Heavy Ion Large area proportional counter Telescope for solar and anomalous cosmic rays, IEEE Trans. Geosci. Remote Sensing 31, $542,1993$.

Mason, G. M., et al., LEICA: A Low Energy Ion Composition Analyzer for the study of solar and magnetospheric ions, IEEE Trans. Geosci. Remote Sensing 31,549, 1993.

McDonald, F. B., et al., The anomalous abundance of cosmic ray nitrogen and oxygen nuclei at low energies, Astrophys. J. 185, L105, 1974.

Mewaldt, R. A., Temporal variations of anomalous cosmic rays and further evidence for anomalous cosmic ray hydrogen, Proc. 21st Internat. Cosmic Ray Conf. (Adelaide) 6, 160, 1990.

Mewaldt, R. A., et al., The return of the anomalous cosmic ray component to $1 \mathrm{AU}$ in 1992, Proc. 23rd Internat. Cosmic Ray Conf. (Calgary) 3, 401, 1993.

Mewaldt, R. A., J. D. Spalding, and E. C. Stone, The isotopic composition of the anomalous low energy cosmic rays, Astrophys. J. 283, 450, 1984.

Mewaldt, R. A., E. C. Stone, and R. E. Vogt, Implications of time variations for the origin of low energy cosmic ray nitrogen and oxygen nuclei, Proc. 14th Internat. Cosmic Ray Conf. (Munich) 2, 804, 1975.

Mogro Campero, A., N. Schofield, and J. A. Simpson, On the origin of low energy heavy nuclei below $\sim 30 \mathrm{MeV}$ per nucleon observed in interplanetary space during quiet times, 1968-72, Proc. 13th Internat. Cosmic Ray Conf. (Denver) 1, 140, 1973.

Pesses, M. E., J. R. Jokipii, and D. Eichler, Cosmic ray drift, shock wave acceleration, and the anomalous component of cosmic rays, Astrophys. J., 246, L85, 1981.

Saito, T., and D. B. Swinson, The inclination of the heliospheric current sheet and cosmic ray intensity at Earth, J. Geophys. Res. 91, 4536, 1986.

Teegarden, B. J., F. B. McDonald, and V. K. Balasubrahmanyan, Spectra and charge composition of the low energy galactic cosmic radiation from $\mathrm{Z}=2$ to 14 , Acta Physica Hungarica 29, Suppl. 1, 345, 1970.

von Rosenvinge, T. T., and F. B. McDonald, IMP 6, 7, and 8 observations of the composition and time variations of low energy cosmic rays, Proc. 14th Internat. Cosmic Ray Conf. (Munich) 2, 792, 1975.

Webber, W. R., E. C. Stone, and R. E. Vogt, The elemental composition of quiet time low energy cosmic rays measured on the Voyager spacecraft, Proc. 16th Internat. Cosmic Ray Conf. (Kyoto) 5, 357, 1977.

A. C. Cummings, J. R. Cummings, R. A. Mewaldt, and E. C. Stone, 220-47 California Institute of Technology, Pasadena CA 91125.

B. Klecker, D. Hovestadt, and M. Scholer, Max-PlanckInstitut fur Extraterrestrische Physik, D-8046 Garching, FRG. G. M. Mason, J. E. Mazur, and D. C. Hamilton, University of Maryland, College Park, MD 20742.

T. T. von Rosenvinge, NASA/Goddard Space Flight Center, Greenbelt MD 20771.

J. B. Blake, Aerospace Corporation, El Segundo CA 90009.

(Received August 12, 1993; accepted August 23, 1993) 